\title{
RACIPE: a computational tool for modeling gene regulatory circuits using randomization
}

Bin Huang ${ }^{1}$, Dongya Jia ${ }^{1,2}$, Jingchen Feng ${ }^{1}$, Herbert Levine ${ }^{1,3,4,5^{*}}$, José N. Onuchic ${ }^{1,4,5,6^{*}}$ and Mingyang Lu ${ }^{7^{*}}$

\begin{abstract}
Background: One of the major challenges in traditional mathematical modeling of gene regulatory circuits is the insufficient knowledge of kinetic parameters. These parameters are often inferred from existing experimental data and/or educated guesses, which can be time-consuming and error-prone, especially for large networks.

Results: We present a user-friendly computational tool for the community to use our newly developed method named random circuit perturbation (RACIPE), to explore the robust dynamical features of gene regulatory circuits without the requirement of detailed kinetic parameters. Taking the network topology as the only input, RACIPE generates an ensemble of circuit models with distinct randomized parameters and uniquely identifies robust dynamical properties by statistical analysis. Here, we discuss the implementation of the software and the statistical analysis methods of RACIPE-generated data to identify robust gene expression patterns and the functions of genes and regulatory links. Finally, we apply the tool on coupled toggle-switch circuits and a published circuit of B-lymphopoiesis.
\end{abstract}

Conclusions: We expect our new computational tool to contribute to a more comprehensive and unbiased understanding of mechanisms underlying gene regulatory networks. RACIPE is a free open source software distributed under (Apache 2.0) license and can be downloaded from GitHub (https://github.com/simonhb1990/ RACIPE-1.0).

Keywords: Random circuit perturbation, RACIPE, Gene regulatory circuits, GRNs, Dynamical features, Statistical analysis

\section{Background}

Biological processes are orchestrated by complex gene regulatory networks (GRNs). To understand the operating principles of GRNs, mathematical modeling approaches $[1,2]$ have been widely used in various contexts, such as regulation of cell cycle [3], stem cell development [4], circadian rhythm [5], developmental pattern formation [6] and cell phenotypic switches in cancer [7-11]. To model the dynamics of GRNs, different computational algorithms have been developed [12], such as ordinary differential equations (ODEs)-based models [13], Boolean network models [14, 15], Bayesian network models [16],

\footnotetext{
* Correspondence: Herbert.Levine@rice.edu; jonuchic@rice.edu;

Mingyang.Lu@jax.org

${ }^{1}$ Center for Theoretical Biological Physics, Rice University, Houston, TX, USA

${ }^{7}$ The Jackson Laboratory, Bar Harbor, ME, USA

Full list of author information is available at the end of the article
}

agent-based models [17], and reaction-diffusion models [18]. The ODEs-based models consider more regulatory details compared to Boolean or Bayesian network models and less computationally intensive than agent-based model and reaction-diffusion models, thus being a very attractive approach to simulate the operation of GRNs. GRN modeling has been integrated with methods to design and optimize the gene circuits in systems and synthetic biology [19-22].

It is believed that there is a core gene regulatory circuit underlying a GRN which functions as a decision-making module for one specific biological process [23, 24]. Identification of such core gene circuits can largely reduce the complexity of network modeling. Notably, the core gene regulatory circuit doesn't function alone. Instead, its operation is usually regulated by other genes and signaling pathways ("peripheral factors") that interact with the core

(c) The Author(s). 2018 Open Access This article is distributed under the terms of the Creative Commons Attribution 4.0 International License (http://creativecommons.org/licenses/by/4.0/), which permits unrestricted use, distribution, and 
circuit. Although the ODE-based and other modeling approach have been successfully applied to analyze the dynamics of the core gene circuits in certain scenarios, these approaches typically suffer from two issues. First, it is very difficult for traditional modeling approach to consider the effects of these "peripheral" factors due to their inherent complexity. Second, the modeling approaches are usually limited by insufficient knowledge of the kinetic parameters for many of the biological processes. In this case, the values of most parameters have to be inferred either by educated guess or fitting to the experimental results, which can be time-consuming and error-prone especially for large gene networks.

To deal with these issues, we previously established a new computational method, named random circuit perturbation (RACIPE), to study the robust dynamical features of gene regulatory circuits without the requirement of detailed kinetic parameters [25]. RACIPE takes the topology of the core regulatory circuit as the only input and unbiasedly generates an ensemble of mathematical models, each of which is characterized by a unique set of kinetic parameters. For each mathematical model, it contains a set of chemical rate equations, which are subjected to non-linear dynamics analysis. From the ensemble of models, we can analyze the robust dynamical properties of the core circuit by statistical analysis. In RACIPE, the effects of the "peripheral factors" are modeled as random perturbations to the kinetic parameters.

Unlike the traditional ODEs-based modeling [26], RACIPE uses a self-consistent scheme to randomize all kinetic parameters for each mathematical model instead of relying on a particular set of parameters. Unlike other methods using randomization [27-30], RACIPE adopts a more carefully designed sampling strategy to randomize parameters across a wide range while satisfying the half-function rule, where each regulatory link has about $50 \%$ chance to be activated in the ensemble of RACIPE models. Also, unlike other methods to estimate parameters of ODEs from the experimental data [31, 32], RACIPE is designed to explore the robust features of the gene regulatory circuits in a much broader ranges of parameters even without the input of experimental data. Then, RACIPE-generated gene expression data and corresponding parameters can be analyzed by statistical learning methods, such as hierarchical clustering analysis (HCA) and principal component analysis (PCA), which provides a holistic view of the dynamical behaviors of the gene circuits. Notably, RACIPE integrates statistical learning methods with parameter perturbations, which makes it distinct from the traditional parameter sensitivity analysis [27, 30], parameter space estimation [31] and other randomization strategies [28, 29]. In addition, our previous work shows that robust gene expression patterns are conserved against large parameter perturbations due to the restraints from the circuit topology. Thus, we can interrogate the dynamical property of a gene circuit by randomization.

Without the need to know detailed kinetic parameters, RACIPE can 1) identify conserved dynamical features of a relatively large gene regulatory circuits across an ensemble of mathematical models; and 2) generate predictions on gain-of-function and loss-of-function mutations of each gene/regulatory link; and 3) discover novel strategies to perturb particular cell phenotypes. The application of RACIPE to a proposed core 22-gene regulatory circuit governing epithelial-to-mesenchymal transition (EMT) showed that RACIPE captures experimentally observed stable cell phenotypes, and the efficiency of various biomarkers in distinguishing different EMT phenotypes [25].

Here, we report a new computational tool that we developed to easily implement the random circuit perturbation method. In the following, we first discuss the implementation of RACIPE, including how the tool processes the input topology file of a gene network, estimates the range of parameters for randomization and solves stable steady states, etc. By applying RACIPE on a coupled toggle-switch circuit, we evaluate the computational cost of using RACIPE, detail the procedure on how to choose an appropriate number of RACIPE models and number of initial conditions for each RACIPE model to get converged simulation results for a gene circuit, and further illustrate how to do perturbation analysis using RACIPE. Lastly, we apply RACIPE on a published gene circuit governing B-lymphopoiesis [33] and show that RACIPE can capture multiple gene expression states during $B$ cell development and the fold-change in expression of several key regulators between stages [34]. In summary, we expect RACIPE will be a valuable and user-friendly tool for the community to decipher the robust dynamical features of gene circuits in many applications.

\section{Implementation}

RACIPE method is developed to identify the robust dynamical features of a biological gene circuit without the need of detailed circuit parameters [25]. RACIPE can generate and simulate an ensemble of models (Fig. 1a) and statistical analysis methods can be used to identify robust features of the circuit across all generated models. Here we report a newly developed tool based on the RACIPE method specifically for multi-stable gene regulatory circuits. With the input of the topology of a gene circuit, the tool automatically builds mathematical models for the circuit, randomizes the model parameters, and calculate the solutions of the stable steady states. These results can be used to uncover the robust features of the circuit, such as the stable steady-state 


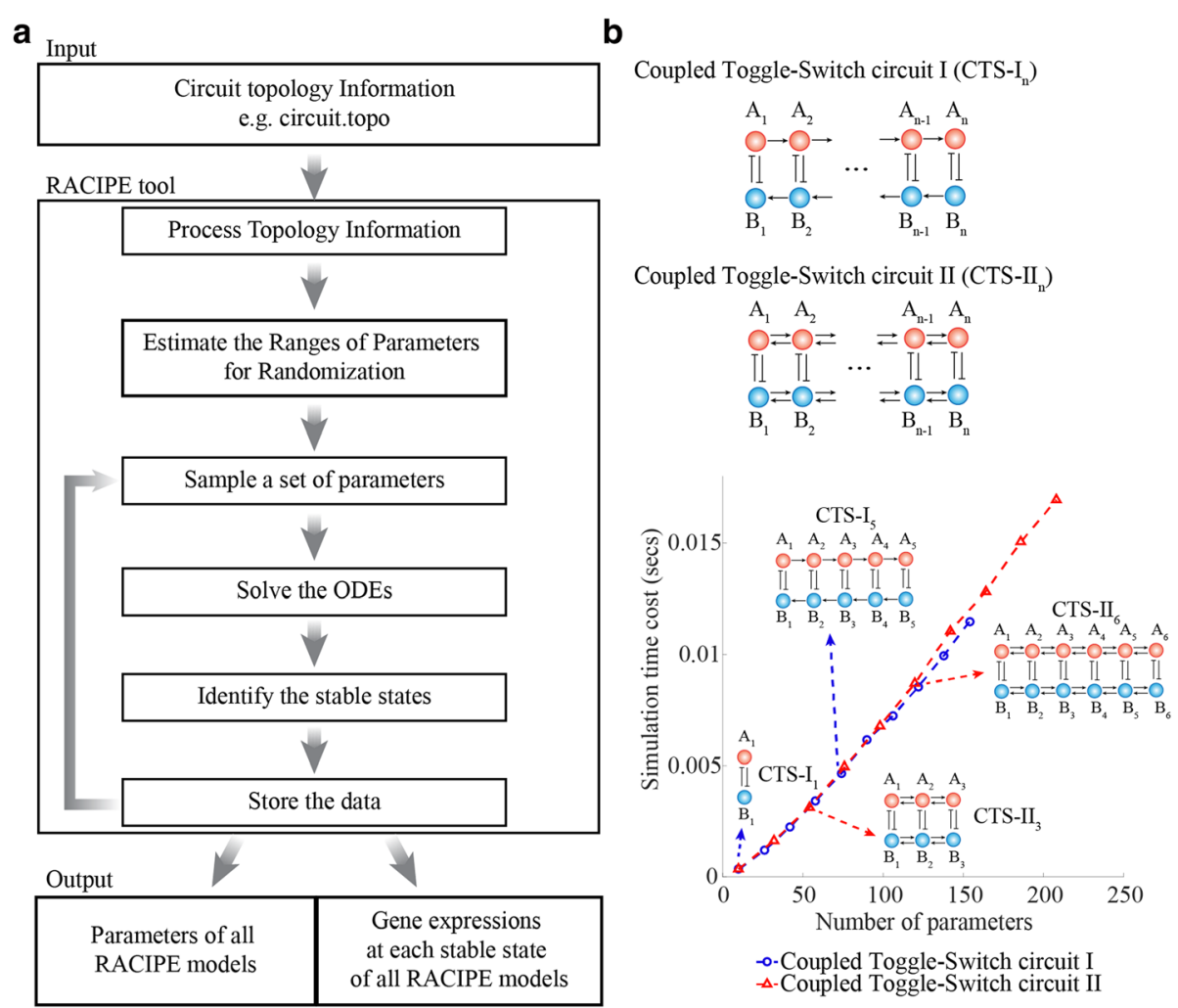

Fig. 1 The computational tool of random circuit perturbation (a) Workflow of RACIPE. The only input for the tool is the circuit topology information. RACIPE automatically estimates the ranges of kinetic parameters for randomization and, from these ranges, randomly samples a particular set of parameters for a model. Then, it simulates the rate equations for this model to find all possible stable states. This procedure is repeated for many times to generate an ensemble of models. Finally, the tool outputs, from all the models, the kinetic parameters and the simulated gene expression of all stable states. $\mathbf{b}$ RACIPE is tested on two types of coupled toggle-switch (CTS) circuits (diagram illustrated in the top panel). The arrows represent transcriptional activation; the bar-headed arrows represent transcriptional inhibition. For both of the cases, the average time cost to simulate a RACIPE model ( $y$-axis) is linearly proportional to the number of model parameters ( $x$-axis)

gene expressions. The RACIPE tool currently can only calculate the solutions for the stable steady states but can be easily extended to study the temporal dynamics of a gene circuit. The main steps of the tool are elaborated below.

\section{Input data}

The main input of RACIPE is the topology of a gene circuit, i.e. the gene names and the regulatory links connecting them. The current version can be applied to gene regulatory circuits with only transcription factors. We will expand its capacity to other regulation types in the future. In the input topology file (e.g., "circuit.topo"), each line specifies a regulatory link, which contains the name of the source gene, the name of the target gene, and the type of interactions (activation or inhibition). The list of gene nodes is not required, as it is automatically generated in RACIPE. Table 1 shows an example of the input topology file for a toggle-switch circuit, which has two mutually inhibiting genes $\mathrm{A}$ and $\mathrm{B}$.

\section{Process circuit topology information}

Based on the input circuit topology, RACIPE automatically builds mathematical models using ordinary differential equations (ODEs). For instance, the temporal dynamics of a toggle switch circuit can be modeled by the following ODEs:

$$
\begin{aligned}
& \dot{A}=G_{A} H^{S}\left(B, B_{A}^{0}, n_{B A}, \lambda_{B A}^{-}\right)-k_{A} A \\
& \dot{B}=G_{B} H^{S}\left(A, A_{B}^{0}, n_{A B}, \lambda_{A B}^{-}\right)-k_{B} B
\end{aligned}
$$

where $A$ and $B$ represent the protein levels of $\mathrm{A}$ and $\mathrm{B}$ encoded by genes A and B, respectively. $G_{A}$ and $G_{B}$ are the

Table 1 Format of the input topology file ("circuit.topo")

\begin{tabular}{lll}
\hline Source & Target & Type $^{\#}$ \\
\hline A & B & 2 \\
B & A & 2 \\
\hline
\end{tabular}

" 1 " stands for activation, and "2" stands for inhibition 
maximum production rates (the production rate with all activators, but not any inhibitor, binding to the promoter region of the targeted gene). $k_{A}$ and $k_{B}$ are the innate degradation rates of the proteins $A$ and $B$, respectively. The effects of the inhibitory regulation of gene $\mathrm{A}$ by $\mathrm{B}$ is formulated as a non-linear shifted Hill function [8] $H^{S}\left(B, B_{A}^{0}\right.$, $\left.n_{B A}, \lambda_{B A}^{-}\right)$defined as

$$
H^{S}\left(B, B_{A}^{0}, n_{B A}, \lambda_{B A}^{-}\right),=\lambda_{B A}^{-}+\left(1-\lambda_{B A}^{-}\right) H^{-}\left(B, B_{A}^{0}, n_{B A}\right)
$$

where $H^{-}=1 /\left(1+\left(B / B_{A}^{0}\right)^{n_{B A}}\right)$ is the inhibitory Hill function, $B_{A}^{0}$ is the threshold level, $n_{B A}$ is the Hill coefficient and $\lambda_{B A}^{-}$is the maximum fold change of the A level caused by the inhibitor $\mathrm{B}\left(\lambda_{B A}^{-}<1\right)$. The inhibition of gene $B$ by gene $A$ can be modeled in a similar way. For gene circuits with excitatory links, the regulation of activation can also be modeled by the shifted Hill function, now with the fold change $(\lambda)$ larger than 1 .

When multiple regulators target a gene, the functional form of the rate equations depends on the nature of the multivalent regulation. Currently, we adopt a common scheme where we assume that these regulatory interactions are independent. Thus, the overall production rate is written as the product of the innate production rate of the target gene and the shifted Hill functions for all the regulatory links. We will consider other cases, such as competitive regulation, in a later version.

\section{Estimate the ranges of parameters for randomization}

Next, RACIPE estimates, for each parameter, the range of values for randomization. Most of the parameter ranges, such as the ones of production and degradation rates, are preset (see Additional file 1: SI 1.1), while the ranges of the threshold values in the shift Hill functions are estimated numerically to satisfy the "half-functional" rule. The "half-functional" rule ensures that each link in the circuit has roughly $50 \%$ chance to be functional across all the models [25]. All the parameter ranges are generated and stored in a parameter file ("circuit.prs").

\section{Solve and identify the stable steady states}

To generate a model, RACIPE randomizes each parameter independently within the pre-calculated range. For each model with a particular set of parameters, RACIPE numerically simulates the dynamics of the model (see Additional file 1: SI 1.2). To identify all possible stable steady states of each model, RACIPE repeats the simulations for multiple times with different initial conditions, randomly chosen from a log-uniform distribution ranging from the minimum possible level to the maximum possible level. The stable steady states can be obtained in
RACIPE by simulating the dynamics using the Euler method or the Runge-Kutta method. From the steady state solutions of all the realizations, we identify distinct stable states, defined as those whose Euclidean distances of the levels among them are all larger than a small threshold (see Additional file 1: SI 1.3). The above procedure is repeated for all the models. Together, we obtain a large set of gene expression data and model parameters for statistical analysis. In the implementation, RACIPE randomly generates a number mathematical of models, each of which is subject to simulations from a number of initial conditions. We will discuss how to appropriately choose the number of RACIPE models and the number of initial conditions for each RACIPE model in the Results section.

\section{Output data}

Lastly, the model parameters and the steady state gene expressions of all RACIPE models are stored separately. The parameters for each RACIPE model are stored in "circuit_parameter.dat", where each row corresponds to one RACIPE model, and each column shows the value of a parameter. The parameters follow the same order in the "circuit.prs" file. Depending on the number of stable states of a RACIPE model, its gene expressions are stored in the "circuit_solution_i.dat", where $\mathrm{i}$ is the number of stable states. In the "circuit_solution_i.dat", each row shows the gene expression vectors of all the stable steady states from a RACIPE model. These data are subject to further statistical analysis.

\section{Options}

RACIPE allows adjusting simulation parameters by directly specifying them in the command line or in the "circuit.cfg" file (see the README file for detailed instructions). RACIPE allows the user to choose different ODE solvers (the first-order Euler or the Runge-Kutta method) and to export any RACIPE model into the SBML format [35, 36]. Moreover, RACIPE also has options to perform simulations of perturbations, such as gene knockout, gene overexpression and knockdown, and removal of a regulatory link. Unlike conventional approach, RACIPE applies perturbations (see Additional file 1: SI 1.4) to the entire ensemble of models to capture the conserved behaviors of the treatment.

\section{Results}

\section{Time cost of simulations}

To evaluate the performance of the tool with different choices of simulation parameters, we test the tool on two types of coupled toggle-switch (CTS) circuits (Fig. 1b, see Additional file 1: SI section "Results" for mathematical models). They both contain several toggle-switch motifs, but different connecting patterns among these motifs, 
where the type I circuits (CTS-I) have unidirectional activations among A genes (B genes), while the type II circuit (CTS-II) have mutual activations among A genes (B genes). These circuits have been actively studied to understand the coupled cellular decision-making processes $[37,38]$. By changing the number of toggle-switch motifs, we can easily test RACIPE on circuits of different sizes. For each circuit, we generate 10,000 random models and solve steady-state expressions starting from 1000 initial conditions for each model. As shown in Fig. $1 \mathrm{~b}$, for both types of circuits, the average simulation time to solve a RACIPE model scales linearly with the total number of parameters in the model, suggesting its potential use on large circuits. Of note, the total time to simulate all RACIPE models depends on other factors (the number of models, the number of initial conditions, etc.), which will be discussed in the next section.

\section{Convergence test}

As mentioned above, there are two important simulation parameters - the number of RACIPE models (nRM) and, for each model, the number of initial conditions (nIC) that are used to find all possible stable steady states. When nRM and nIC are too small, the results from the ensemble of models may not converge and be statistically significant. However, having too large nRM and nIC sacrifices computational efficiency.

To identify an optimal choice of nRM and nIC, we test the effects of both on the convergence of the simulation results by calculating the dissimilarity of the probability distribution of the number of stable states (referred to as the "dissimilarity of states") and the distribution of gene expressions (referred to as the "dissimilarity of expressions") using different values of nRM and nIC (Figs. 2 and 3). If the simulation results converge well, the dissimilarity values are expected to be small.

For every choice of nIC and nRM, we repeat the RACIPE calculations for ten times for each circuit and measure the dissimilarity of the above-mentioned probability distributions by the Bhattacharyya distance [39] $D_{B}=-\ln \left(\sum_{x \in X} \sqrt{p(x) q(x)}\right.$, where $p$ and $q$ are two distributions. If the two distributions are exactly same, $D_{B}$ equals to 0 ; The more different the two distributions are, the larger $D_{B}$ becomes. We have also calculated the dissimilarity using a different distance metric (the Kullback-Leibler divergence [40]) and obtained similar results (Additional file 1: Figure S9).

To explore the effects of nRM on the distribution of the number of stable states, we repeat RACIPE on the circuit for ten times for a certain nRM, and calculate the distribution of the number of stable states for each replica. Then we compare the dissimilarity of the distributions (i.e. the dissimilarity of states) for different nRMs by calculating the average Bhattacharyya distances:

$$
D_{B}=\frac{1}{100} \sum_{j=1}^{10} \sum_{h=1}^{10}-\ln \left(\sum_{x \in X} \sqrt{p_{n_{i}}(x) p_{n_{m} h}(x)}\right)
$$

where $p_{n_{i}}(x)$ stands for the probability of the circuit with $x$ number of stable states for a random model for a replica $j$ when nRM equals to $n_{i} . n_{m}$ is the maximum nRM used in the test. Here, we fix $n_{m}$ to 10,000 . Similarly, we can explore the effects of nRM on the distribution of gene expressions. Similar approach is used to analyze the effects of nIC.

As shown in Fig. 2a and Additional file 1: Figures S3 and S4, the dissimilarity of states decreases when more initial conditions are used. When nIC is larger than 500, RACIPE can effectively identify most stable steady states, except for some rare states (the probability to be observed is less than $1 \%$ ). To get converged distribution of the number of stable states, the minimum required nIC increases with the size of the circuit (Fig. 2b and Additional file 1: Figure S3). Surprisingly, the convergence of the distribution of expressions seems to be less sensitive to nIC (Fig. 2c and Additional file 1: Figure S5 and S6), as similar results are obtained no matter how small or larger nICs are selected. As suggested from Fig. 2d, with more than 10,000 RACIPE models, 100 initial conditions are sufficient to get converged results.

However, nRM has a significant influence on the convergence of the simulation results. From Fig. 2a and Additional file 1: Figure S4, increasing nRM dramatically lowers the dissimilarity of states. Also, without enough RACIPE models, the distribution of expressions does not converge even when a large nIC is used (Fig. 2d). Furthermore, when nIC equals to 1000 , both the dissimilarity of states and gene expressions decrease when nRM increases (Fig. $3 a, b$ and Additional file 1: Figure S8). To get converged results for the distribution of states, the minimum required $\mathrm{nRM}$ again increases with the size of the circuit (Fig. 3c and Additional file 1: Figure S10). However, the minimum required nRM to get the converged distribution of expressions is likely independent to the size of the circuit as long as it is more than 7000 (Fig. 3d). Interestingly, when the dissimilarities of states for different circuits are scaled by the maximum number of stable states of the circuits, the curves of the dissimilarities for each circuit overlap with each other (Additional file 1: Figure $\mathrm{S} 8 \mathrm{~b}$ ). The results suggest that the higher dissimilarity of a larger circuit is due to the higher complexity of the system. 

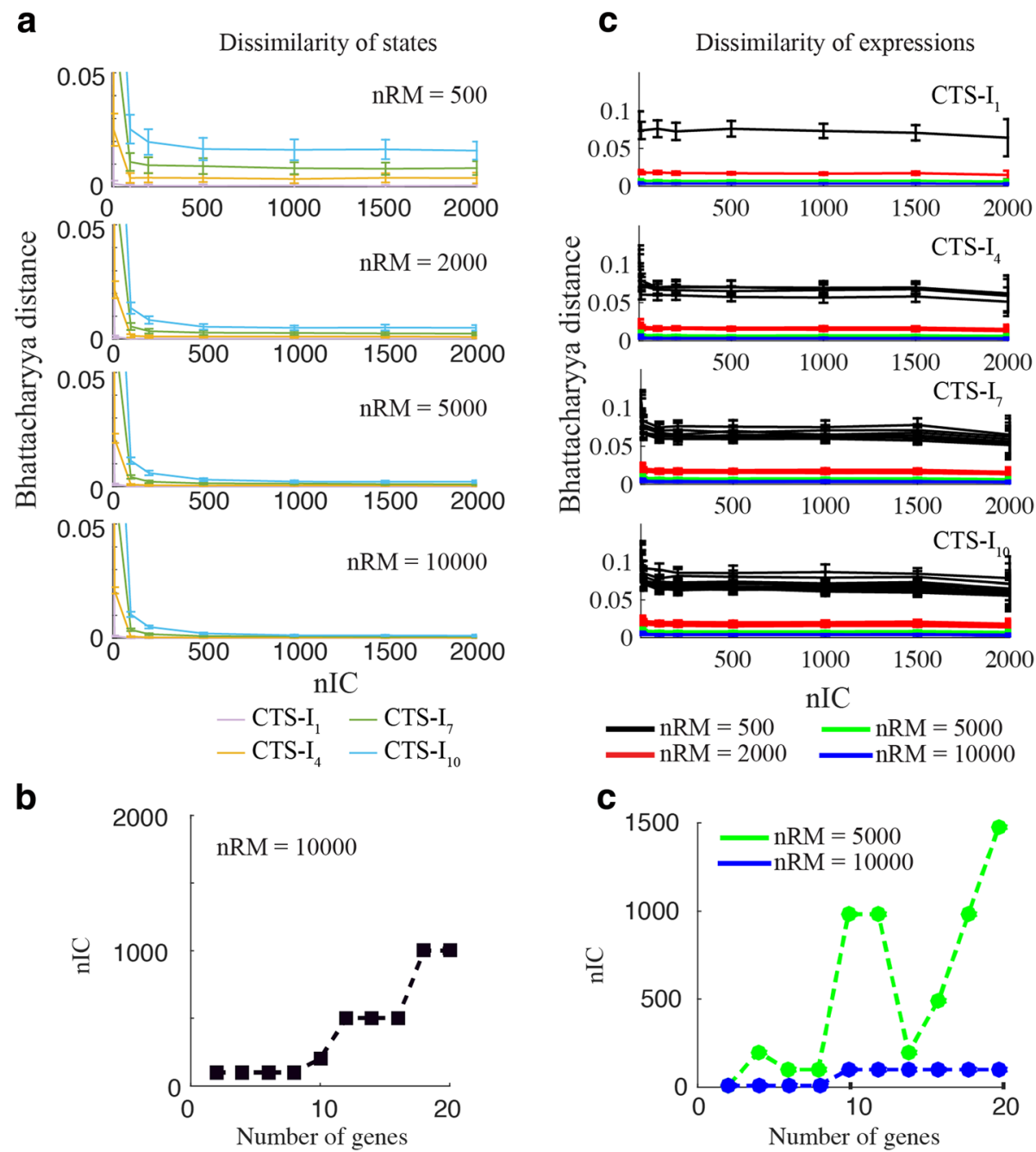

Fig. 2 The effect of the number of initial conditions on the convergence of the RACIPE results. a For each coupled toggle-switch I (CTS-I) circuit (curves in different colors), the convergence is evaluated by the dissimilarity of states using different numbers of initial conditions (nIC in $x$-axis) and different numbers of RACIPE models (nRM in different panels). $\mathbf{b}$ The minimum nIC to get the converged distribution of the number of stables states when nRM equals 10,000. Different points represent the CTS-I circuits of different sizes. The minimum nIC is selected if the decrease of the Bhattacharyya distance is smaller than the threshold (0.0005, see Additional file 1: Figure S3) when nIC increases. c For each CTS-I circuit, the convergence is alternatively evaluated by the dissimilarity of expressions of each gene. Only the Ai genes for each circuit are plotted (one line per gene) and colored differently for different nRMs. The dissimilarity is less sensitive to nIC, but is dramatically reduced with the increase of nRM. $\mathbf{d}$ The minimum $\mathrm{nIC}$ to get the converged distribution of expressions. The minimum nIC is selected if the decrease of the Bhattacharyya distance is smaller than the threshold (0.0005, see Additional file 1: Figure S6) when nIC increases. nRM needs to be larger than 5000 , otherwise the distribution is not converged even with $\mathrm{nIC}=2000$

\section{Analysis of the RACIPE-generated data}

Once RACIPE generates, for each model, the kinetic parameters and the stable-state gene expressions, a variety of statistical methods can be applied to analyze the data from the ensemble of models. In the following, we will illustrate these analyses in the context of a coupled toggle-switch circuit (CTS-I ${ }_{5}$, with five toggle switches) (Fig. 4a). We generate 10,000 RACIPE models, each of which is simulated starting from 1000 initial conditions. For each model, the maximum number of stable steady states is seven (Additional file 1: Figure S2); from 10,000
RACIPE models, there is a total of 24,425 steady states. These states could be regarded as the gene expressions of cells in a system obeying these dynamics.

To analyze the simulated gene expression, RACIPE utilizes average linkage hierarchical clustering analysis (HCA) using Euclidean distance after normalization of the expressions (see Additional file 1: SI 1.5-1.8 for details). From the heatmap (Fig. 4b), we observe six major clusters each of which has at least $5 \%$ fraction (Fig. 4c). The six major clusters, denoted by "gene states" below, are further confirmed by projecting all steady 

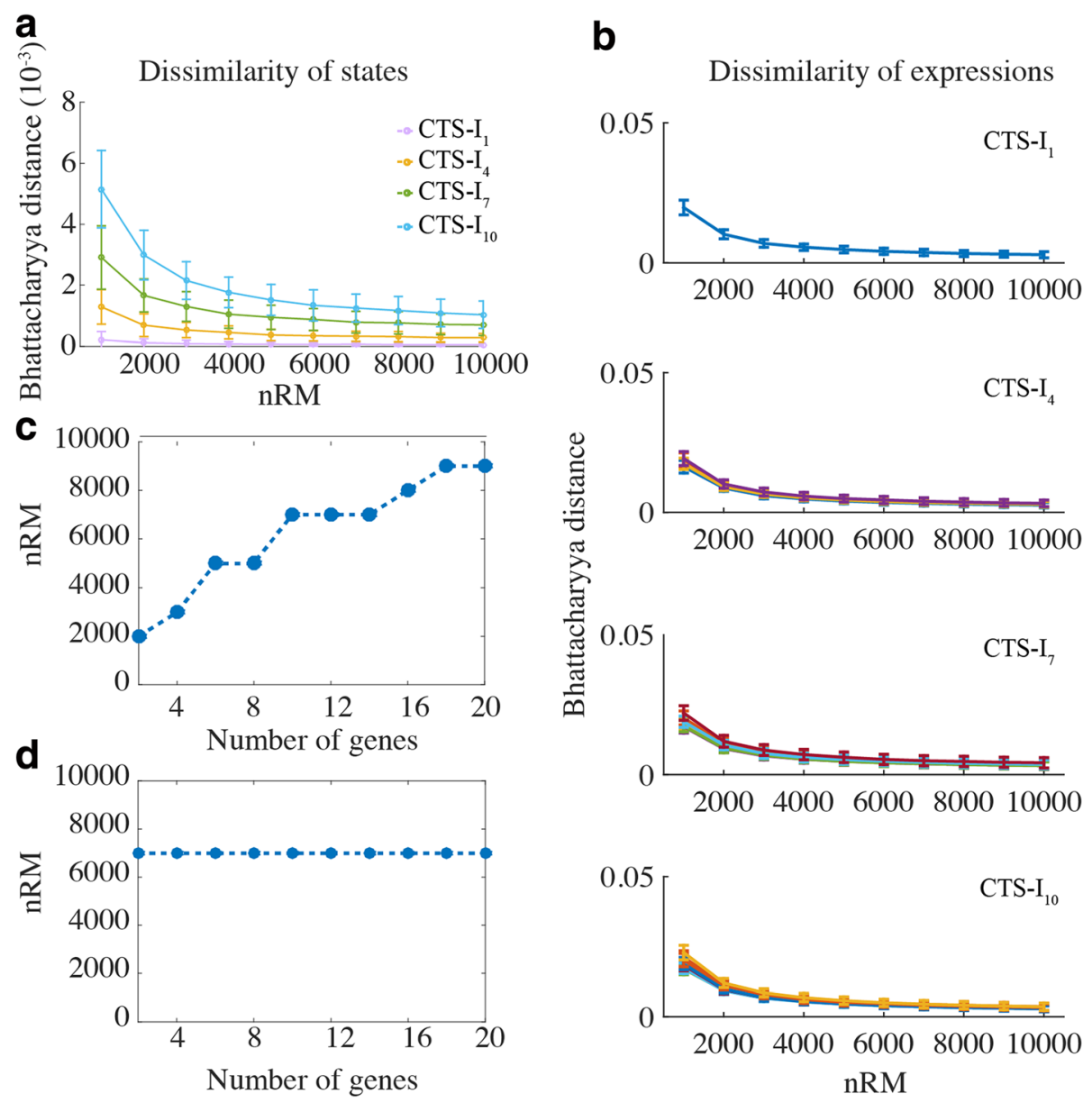

Fig. 3 The effect of the number of RACIPE models on the convergence of the results. a The dissimilarity of states as a function of nRM when nIC is 1000. $\mathbf{b}$ The dissimilarity of expressions as a function of nRM when $\mathrm{nIC}$ is 1000 . $\mathbf{c}$ The minimum nRM as the function of the number of genes in each circuit. $\mathbf{d}$ The minimum nRM to get the converged distribution of gene expressions

state solutions onto the first two principal components (PC1 and PC2) (Fig. 4d). From HCA, genes with similar functions are also grouped together. Strikingly, the gene expression patterns of the couple toggle-switch circuits, from the top to the bottom, correspond to a cascade of flips of the state of each toggle-switch motif (Fig. 4b). For instance, compared with gene state 2 , gene state 5 has a flipped state in the fifth toggle-switch motif $\left(\mathrm{A}_{5}\right.$ and $\mathrm{B}_{5}$ ).

Moreover, RACIPE can identify the roles of individual genes in the dynamic behaviors of the circuit by in silico gene knockouts, one gene at a time (Fig. 5 and Additional file 1: Figure S13). Knocking out gene $A_{1}$ dramatically changes the probability distribution of the number of stable states and probability distribution of gene expressions, while knocking out gene $\mathrm{A}_{5}$ leads to a similar distribution of the number of stable states and only one gene state is missing. Therefore, we find that, for coupled toggle-switch circuits, the importance of $A_{i}$ genes gradually decreases - $A_{1}$ is the most critical one and $A_{5}$ is the least important one. Similarity, the importance of $B_{i}$ genes is in the reverse order. In addition, RACIPE can identify the significantly differentiated parameters between two states by the statistical analysis of model parameters (Additional file 1: Figures S14, see SI 1.9), which further helps to elucidate the functions of gene circuits.

\section{Application to a B-lymphopoiesis gene circuit}

The above example, while instructive, is only based on simple circuit motifs. To further evaluate the use of RACIPE, we analyze the properties of a gene regulatory circuit governing B-lymphopoiesis. This circuit was previously proposed by Salerno et al. [33] and analyzed mainly by traditional nonlinear dynamics methods, such as bifurcation analysis. Here we compare the RACIPE-generated gene expression data with microarray gene expression profiles of B cells from the previously published work by van Zelm et al. [34].

$\mathrm{B}$ cells that develop in the bone marrow progress through the multipotent progenitor (characterized by 
a

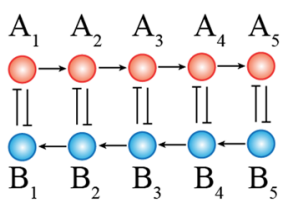

C
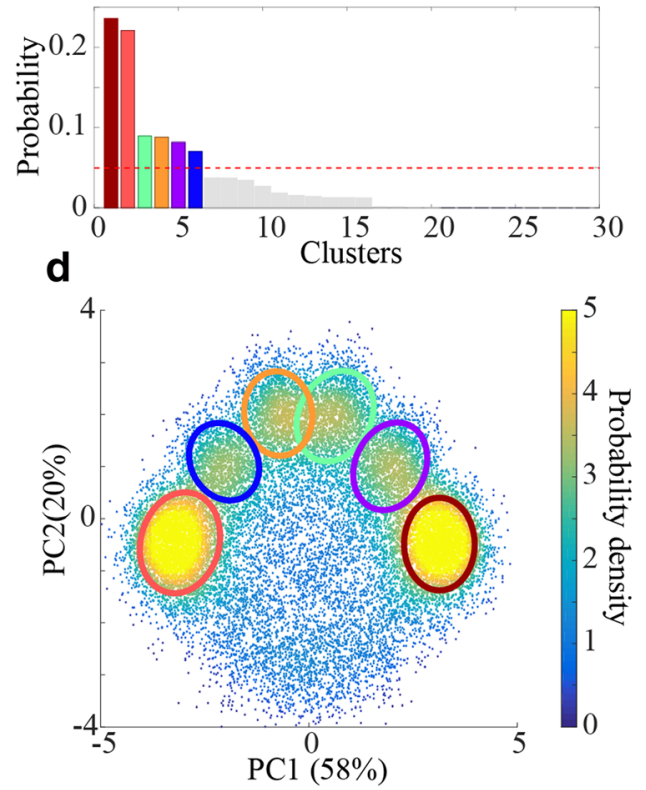

b
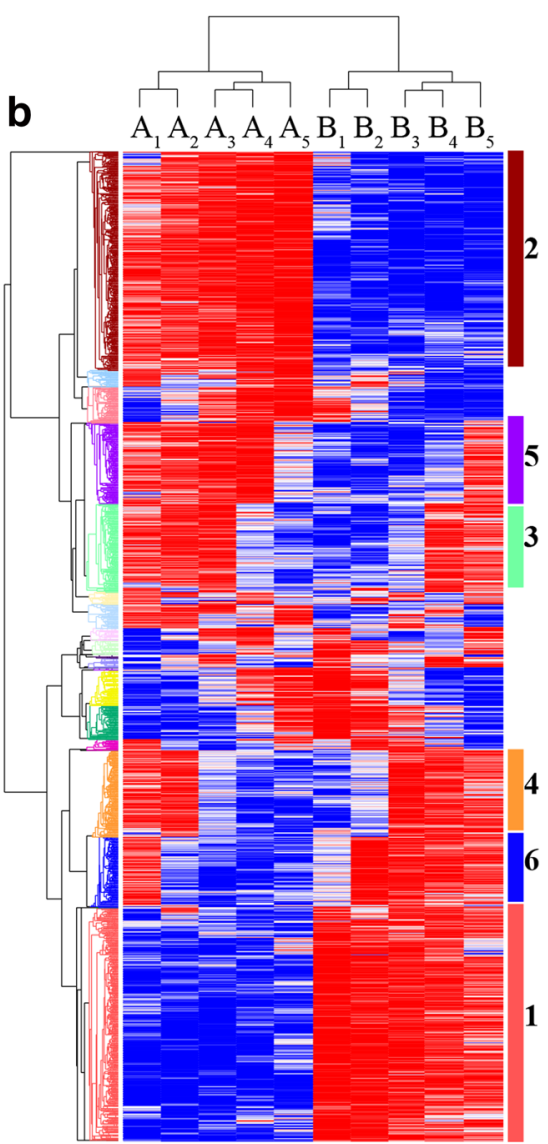

Fig. 4 RACIPE identifies robust gene states of a coupled toggle-switch (CTS-15) circuit. a Diagram of the CTS-I ${ }_{5}$ circuit. b Average linkage hierarchical clustering analysis of simulated gene expressions reveals six major clusters of distinct expression patterns. Each column corresponds to a gene, and each row corresponds to a stable steady state from a RACIPE model. c. Histogram of the fraction of gene expressions in each cluster. The cutoff is selected at 5\% (Red dash line). d 2D probability density map of the RACIPE-generated gene expression data projected on to the first two principal components. The six gene clusters are highlighted by the same colors as those in (b)

$\mathrm{CD}^{+} 4^{+}$lin $^{-}$), pro-B, pre-B-I and pre-B-II large, pre-B-II small and immature-B stages sequentially [34]. The regulatory circuitry for lineage specification of hematopoietic multipotent progenitors is still not well understood. To address this issue, Salerno et al. constructed a gene regulatory circuit (Fig. 6a) governing B-lymphopoiesis based on literature search and confirmed the important role of ZNF521 (zinc finger protein 521) and EBF1 (Early B-Cell Factor 1) during the specification of $B$ cells from the multipotent progenitor stage $\left(\mathrm{CD} 34^{+} / \mathrm{lin}^{-}\right)$to the pro-B stage [33]. Here, we apply RACIPE to the same gene circuit and study the predicted gene expression patterns and how they are associated with various stages during B cell development.

Additional file 1: Figure S15 shows 10,000 models are good enough to capture the robust behaviors of the gene network for B-lymphopoiesis. The stable steady states from all models form four major clusters, which correspond to the stages $\mathrm{CD}_{4} 4^{+} / \mathrm{lin}^{-}$, pro-B, (pre-B-I, Immature-B) and (Pre-B-II large, small), respectively (Fig. 6b-d). We further compare the microarray gene expression profiles with data generated by RACIPE. Even through there is only one sample in each stage from [34], the trend of the gene expression predicted by RACIPE agrees well with that from experiments, especially the comparison between cluster 1 and the $\mathrm{CD}_{4} 4^{+} /$lin $^{-}$stage and that between cluster 3 and the Pre-B-I stage (Fig. 6e). From the hierarchical clustering analysis (Fig. 6b), we observe that there is a 'switch-like' change in the gene expression pattern from the stage pro- $\mathrm{B}$ to pre-B-I, as also shown in Fig. 6c. To test the prediction, we extract the microarray data of pro-B and pre-B-I and analyze the fold-change of the regulators in the circuit. Strikingly, the microarray data show the down-regulation of TF ZNF521, FLT3, IL7Ra and PU.1 and up-regulation of CD19, E2A, PAX5 and EBF1, which validates the prediction from the RACIPE analysis (Fig. 6f). In summary, RACIPE is able to provide a rich source of information from the regulatory circuit of B-lymphopoiesis and potentially capture the gene expression features of various stages during $\mathrm{B}$ cell development. 


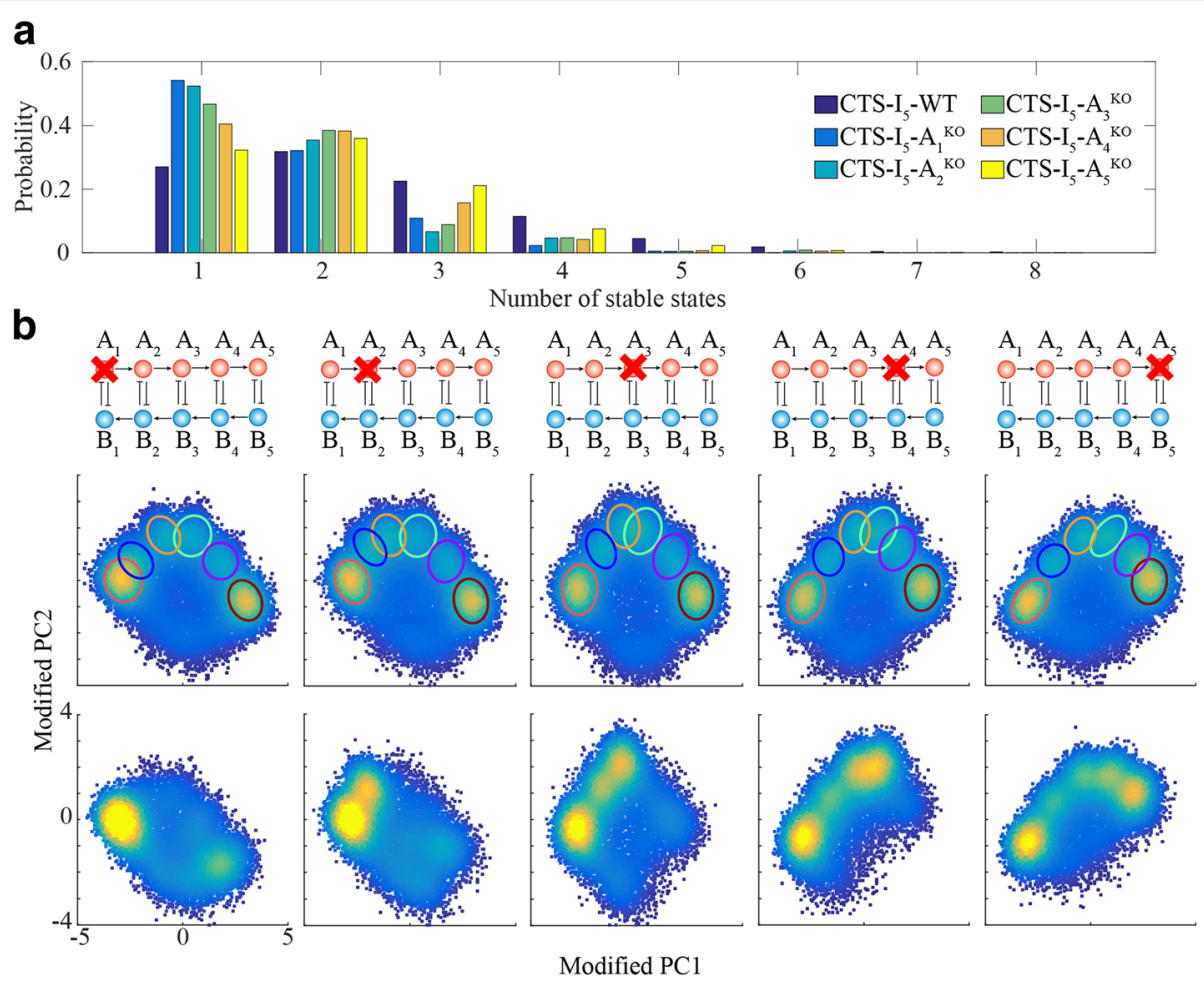

Fig. 5 Perturbation analysis. a Probability distribution of the number of stable steady states of each model. Different colors represent the results of the complete circuit (CTS-I - WT) and different knockout versions $\left(C T S-I_{5}-A_{i}{ }^{K O}\right)$ analyzed by RACIPE. b Probability density maps of the RACIPE gene expressions projected on to the first two principal components. Note, for the knockout cases, the principal components are modified to reflect the zero expressions for the corresponding genes (see SI for details)

Although we observe agreement between in silico clusters by RACIPE and microarray data of various stages in B cell development, we might not yet be able to generate all information regarding the paths of $\mathrm{B}$ cell development. The reasons are at least two-fold. First, the result by RACIPE is highly dependent on the topology of the gene circuit and there might be important genes/regulations missing in the current circuit due to insufficient knowledge from available data. Second, due to the very limited number of experimental samples, i.e., one in each stage, the comparison with clusters by RACIPE might be inaccurate. However, with even the limited information, RACIPE has been shown to capture the change of multiple master regulators across various stages during B cell development. Further studies including construction of a more complete regulatory circuit for B cell development and measures of gene expression of more samples at various stages are needed to fully understand the state transitions of B cell progression.

\section{Discussion}

In this study, we introduced a new tool based on our recently developed computational algorithm, named random circuit perturbation (RACIPE). The tool is built in $\mathrm{C}$ and will be freely available for public use.
Compared to the randomization approaches to generate benchmark datasets for network inference [41, 42], RACIPE features a unique "half-functional" rule to carefully sample the parameter space. In addition, RACIPE can identify the most robust features of a gene circuit, such as gene expression clusters, without the need to know detailed values of kinetic parameters.

To better understand the performance of RACIPE, we particularly explored the effects of two key simulation parameters, the number of initial conditions (nIC) and the number of RACIPE models (nRM), on the convergence of the statistical analysis. Insufficient nIC and nRM may lead to inconsistent results in the repeats of the same simulation. Figs. 2 and 3 are good references for an initial guess of these parameters and users can always identify the optimal nIC and nRM with a similar analysis. From our tests, the time cost of the RACIPE tool scales linearly with the total number of parameters used in the mathematical model, suggesting its potential use in analyzing large gene networks.

To illustrate the use of RACIPE, we applied it to a coupled toggle-switch (CTS-I $\mathrm{I}_{5}$ ) circuit consisting of five toggle switches, a circuit that has an implication in coupled decision-making of multiple cell fates. From the RACIPE-generated expression data, we identified six 

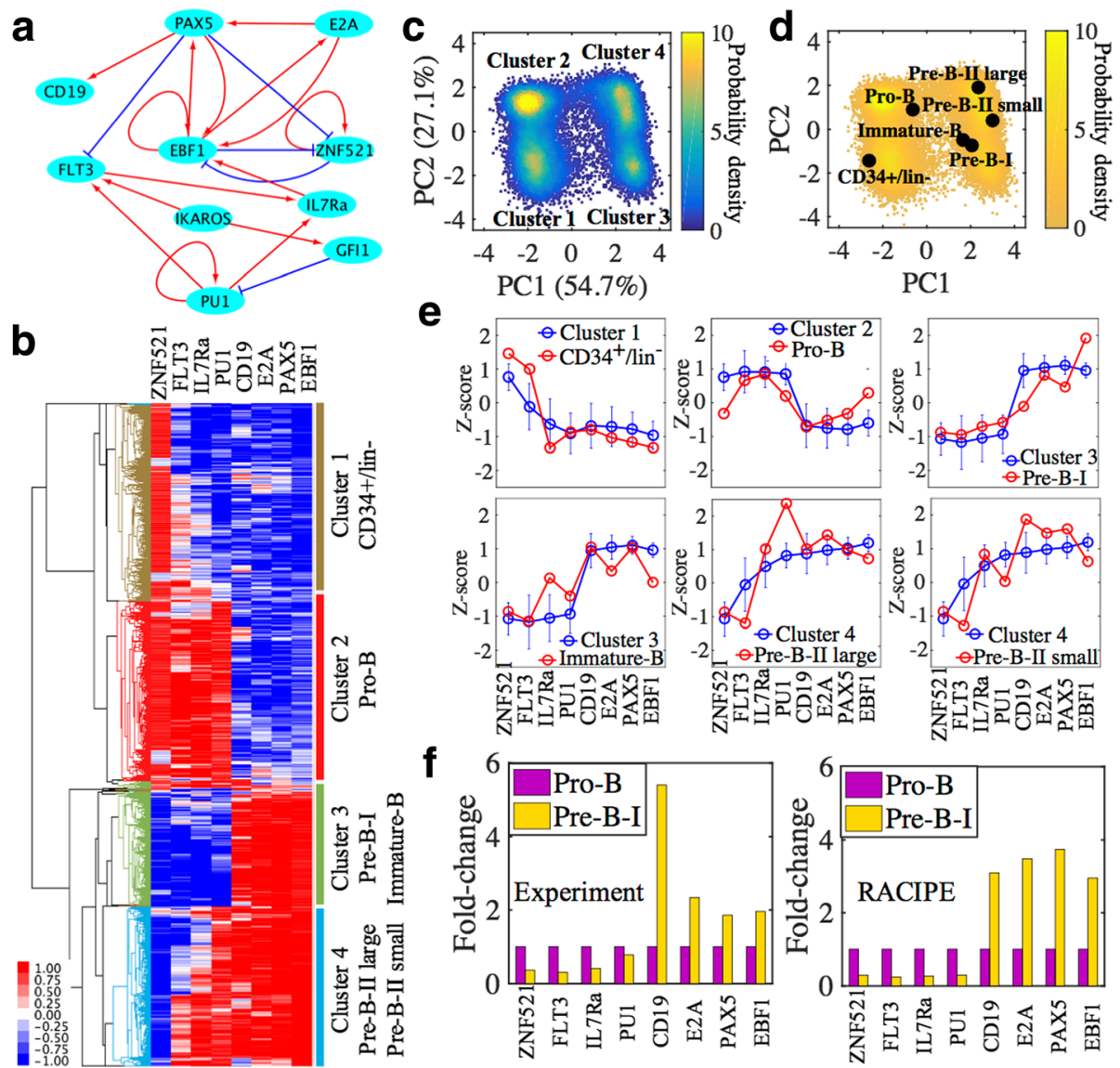

Fig. 6 RAICPE identifies multiple gene expression states during B cell development. a A proposed gene regulatory circuit governing Blymphopoiesis, adopted from (Salerno et al., 2015). The network consists of 10 transcription factors (TFs). Red arrows represent transcriptional activation and blue bar-headed arrows represent transcriptional inhibition. b Average linkage hierarchical clustering analysis of the gene expression data from all the RACIPE models using the Euclidean distance. Each column corresponds to a gene, and each row corresponds to a stable steady state. Four major gene states (clusters) are identified. c 2D probability density map of the RACIPE-predicted gene expression data projected on to the first two principal component axes. $\mathbf{d}$ The microarray expression profiling of different stages during B cell development (van Zelm et al., 2005) projected on to the same axes as shown in (c) (See Additional file 1: SI 1.10). e Comparison between experimental gene expression of various stages with in silico clusters. Blue dots and red dots represent the Z-scores of genes from the RACIPE models and experiments, respectively. Error bar for each blue dot represents standard deviation of the RACIPE-generated gene expression values. $\mathbf{f}$ Comparison between experimental gene expression fold-change from stage Pro-B to stage Pre-B-I with the computed fold-change by RACIPE

major clusters by both HCA and PCA. In addition, we analyzed the role of each gene on circuit dynamics by in silico gene knockout (Fig. 5). To further show the predictive power of RACIPE, we applied it on a published B-lymphopoiesis gene regulatory circuit. The gene expression patterns of various stages during B cell development can be efficiently captured by RACIPE. Notably, the fold-change of master regulators from stage 'Pro-B' to stage 'Pre-B-I' predicted by RACIPE agrees well with that from the microarray data. These results show that RACIPE can not only reveal robust gene expression patterns, but also help uncover the design principle of the circuit.

The capability of RACPE in identifying circuit functions using a randomization approach reinforces the hypothesis that circuit dynamics are mainly determined by circuit topology [43] not by detailed kinetic parameters. Indeed, it is commonly believed that, through evolution, gene circuits of important pathways should be robustly designed to be functional [14] even in a dynamic and heterogeneous environment [44]. In RACIPE, we take advantage of this feature to interrogate the robustness of a gene circuit by randomly perturbing all the kinetic parameters, from which we evaluate the most conserved properties.

Although we believe RACIPE has wide applications in systems biology, there are a few limitations of the current version. First, while all parameters are completely randomized to generate models, some of these models might not be realistic because some parameters are unlikely to be perturbed in cells, such as the number of binding sites. In these cases, incorporating relevant experimental evidences will improve the modeling. Second, RACIPE is 
unique in generating data of both gene expression and model parameters. Although we have shown that the parameters in models from different gene state clusters are distinct (Additional file 1: Figure S14), further data analysis methods are needed to fully understand the roles of each parameter in circuit behavior. Third, the current RACIPE only models regulatory circuits of transcription factors. However, the same approach can be extended to model biological pathways, which typically involves multiple types of regulation, such as protein-protein interactions and microRNA-mediated regulations. Fourth, we currently use deterministic ODE-based method to simulate the circuit dynamics. Since gene expression noise has been shown to play crucial roles in circuit dynamics $[45,46]$, it is important to extend the method to stochastic analysis. Lastly, the quality of the circuit topology may dramatically impact the quality of RACIPE modeling. An accurate inference method for constructing gene circuits is especially important. To associate the parameters with network dynamics, a global sensitivity analysis $[47,48]$ and hyperparameter optimization will be especially useful to measure the effects of each parameter and fit RACIPE models into real gene expression data. Further improvements on these aspects will greatly improve the usability of this randomization-based approach and contribute to a better understanding of the operative mechanisms of gene regulatory circuits.

\section{Conclusions}

In this paper, we have presented a new computational tool based on our recently developed computational method, RACIPE. By taking the topology of GRNs as the only inputs, RACIPE can unbiasedly generate an ensemble of mathematical models, each of which is characterized by a unique set of parameters. We evaluated the convergence of RACIPE-generated results by tuning two simulation parameters - number of initial conditions (nIC) and number of RACIPE models (nRM). In addition, we applied RACIPE on the coupled toggle-switch circuits and a published B-lymphopoiesis network to illustrate the statistical methods that can be applied to RACIPEgenerated data. All told, we expect RACIPE to pave a new way for the community to explore the robust functions of gene regulatory circuits with the insufficient knowledge of the kinetic parameters.

\section{Availability and requirements}

Project name: RACIPE.

Project home page: https:/github.com/simonhb1990/

RACIPE-1.0

Operating system(s): Platform independent.

Programming language: $\mathrm{C}$.

Other requirements: None.
License: Apache 2.0.

Any restrictions to use by non-academics: None.

\section{Additional file}

Additional file 1: Supplementary algorithmic details and user guide of RACIPE and supplementary figures. (DOCX $6198 \mathrm{~kb}$ )

\section{Abbreviations}

CTS: Coupled toggle-switch; EMT: Epithelial-to-mesenchymal transition;

GRN: Gene regulatory network; HCA: Hierarchical clustering analysis; nIC: Number of initial conditions; nRM: Number of RACIPE models; ODE: Ordinary differential equation; PCA: Principal component analysis; RACIPE: Random circuit perturbation

\section{Funding}

This work was supported by the Physics Frontiers Center National Science Foundation (NSF) grant PHY-1427654 and the NSF grants DMS-1361411 and PHY-1605817, and John S. Dunn Foundation Collaborative Research Award (to Jose Onuchic). Mingyang Lu is supported by the start-up fund from the Jackson Laboratory and the National Cancer Institute of the National Institutes of Health under Award Number P30CA034196.

\section{Authors' contributions}

$\mathrm{ML}, \mathrm{HL}$, JNO conceived the study. $\mathrm{BH}, \mathrm{DJ}, \mathrm{JF}, \mathrm{ML}$ performed the research. $\mathrm{BH}$, DJ, ML wrote the manuscript. HL and JNO improved the draft. All authors read and approved the final manuscript.

Ethics approval and consent to participate

Not applicable.

\section{Competing interests}

The authors declare that they have no competing interests.

\section{Publisher's Note}

Springer Nature remains neutral with regard to jurisdictional claims in published maps and institutional affiliations.

\section{Author details}

${ }^{1}$ Center for Theoretical Biological Physics, Rice University, Houston, TX, USA. ${ }^{2}$ Program in Systems, Synthetic and Physical Biology, Rice University, Houston, TX, USA. ${ }^{3}$ Department of Bioengineering, Rice University, Houston, TX, USA. ${ }^{4}$ Department of Biosciences, Rice University, Houston, TX, USA. ${ }^{5}$ Department of Physics and Astronomy, Rice University, Houston, TX, USA. ${ }^{6}$ Department of Chemistry, Rice University, Houston, TX, USA. ${ }^{7}$ The Jackson Laboratory, Bar Harbor, ME, USA.

Received: 14 December 2017 Accepted: 31 May 2018

Published online: 19 June 2018

\section{References}

1. Smolen P, Baxter DA, Byrne JH. Mathematical modeling of gene networks. Neuron. 2000;26:567-80.

2. Novère NL. Quantitative and logic modelling of molecular and gene networks. Nat Rev Genet. 2015;16 nrg3885

3. Tyson JJ. Modeling the cell division cycle: cdc2 and cyclin interactions. Proc Natl Acad Sci U S A. 1991;88:7328-32

4. Huang S, Guo Y-P, May G, Enver T. Bifurcation dynamics in lineagecommitment in bipotent progenitor cells. Dev Biol. 2007;305:695-713.

5. Smolen P, Baxter DA, Byrne JH. Modeling circadian oscillations with interlocking positive and negative feedback loops. J Neurosci. 2001;21:6644-56.

6. Reeves GT, Muratov CB, Schüpbach T, Shvartsman SY. Quantitative models of developmental pattern formation. Dev Cell. 2006;11:289-300.

7. Ao P, Galas D, Hood L, Zhu X. Cancer as robust intrinsic state of endogenous molecular-cellular network shaped by evolution. Med Hypotheses. 2008;70:678-84.

8. Lu M, Jolly MK, Levine H, Onuchic JN, Ben-Jacob E. MicroRNA-based regulation of epithelial-hybrid-mesenchymal fate determination. Proc Natl Acad Sci. 2013;110:18144-9. 
9. Zhang J, Tian X-J, Zhang H, Teng Y, Li R, Bai F, et al. TGF- $\beta$-induced epithelial-to-mesenchymal transition proceeds through stepwise activation of multiple feedback loops. Sci Signal. 2014;7 ra91-ra91

10. Li C, Wang J. Quantifying the landscape for development and Cancer from a Core Cancer stem cell circuit. Cancer Res. 2015;75:2607-18.

11. Yu L, Lu M, Jia D, Ma J, Ben-Jacob E, Levine H, et al. Modeling the genetic regulation of Cancer metabolism: interplay between glycolysis and oxidative phosphorylation. Cancer Res. 2017;77:1564-74.

12. Dehmer M, Emmert-Streib F, Graber A, Salvador A. Applied statistics for network biology: methods in systems biology. In: John Wiley \& Sons; 2011.

13. Strogatz SH. Nonlinear dynamics and chaos. Sarat Book House; 2007.

14. Li F, Long T, Lu Y, Ouyang Q, Tang C. The yeast cell-cycle network is robustly designed. Proc Natl Acad Sci U S A. 2004;101:4781-6.

15. Steinway SN, Zañudo JGT, Ding W, Rountree CB, Feith DJ, Loughran TP, et al. Network modeling of TGF $\beta$ signaling in hepatocellular carcinoma epithelial-to-mesenchymal transition reveals joint sonic hedgehog and Wnt pathway activation. Cancer Res. 2014;74:5963-77.

16. Zhang B, Gaiteri C, Bodea L-G, Wang Z, McElwee J, Podtelezhnikov AA, et al. Integrated systems approach identifies genetic nodes and networks in lateonset Alzheimer's disease. Cell. 2013;153:707-20.

17. Robertson-Tessi M, Gillies RJ, Gatenby RA, Anderson ARA. Impact of metabolic heterogeneity on tumor growth, invasion, and treatment outcomes. Cancer Res. 2015;75:1567-79.

18. Roberts E, Stone JE, Luthey-Schulten Z. Lattice microbes: high-performance stochastic simulation method for the reaction-diffusion master equation. J Comput Chem. 2013;34:245-55.

19. Rodrigo G, Carrera J, Jaramillo A. Genetdes: automatic design of transcriptional networks. Bioinforma Oxf Engl. 2007;23:1857-8.

20. Batt G, Belta C, Weiss R. Model Checking Genetic Regulatory Networks with Parameter Uncertainty. Hybrid Syst Comput Control [Internet]. Springer, Berlin, Heidelberg; 2007 [cited 2018 Mar 26]. p. 61-75. Available from: https://link.springer.com/chapter/10.1007/978-3-540-71493-4_8

21. Beal J, Lu T, Weiss R. Automatic compilation from high-level biologicallyoriented programming language to genetic regulatory networks. PLoS One. 2011;6:e22490

22. Wu F, Su R-Q, Lai Y-C, Wang X. Engineering of a synthetic quadrastable gene network to approach Waddington landscape and cell fate determination. elife. 2017:6:e23702.

23. Milo R, Shen-Orr S, Itzkovitz S, Kashtan N, Chklovskii D, Alon U. Network motifs: simple building blocks of complex networks. Science. 2002;298:824-7.

24. Hartwell LH, Hopfield JJ, Leibler S, Murray AW. From molecular to modular cell biology. Nature. 1999;402:C47-52.

25. Huang B, Lu M, Jia D, Ben-Jacob E, Levine H, Onuchic JN. Interrogating the topological robustness of gene regulatory circuits by randomization. PLoS Comput Biol. 2017;13:e1005456

26. An Introduction to Syst Biol: Design Principles of Biological Circuits [Internet]. CRC Press. 2006 [cited 2017 Dec 11]. Available from: https://www. crcpress.com/An-Introduction-to-Systems-Biology-Design-Principles-ofBiological-Circuits/Alon/p/book/9781584886426

27. Feng X-J, Hooshangi S, Chen D, Li G, Weiss R, Rabitz H. Optimizing genetic circuits by global sensitivity analysis. Biophys J. 2004;87:2195-202.

28. Gutenkunst RN, Waterfall JJ, Casey FP, Brown KS, Myers CR, Sethna JP. Universally sloppy parameter sensitivities in systems biology models. PLoS Comput Biol. 2007:3:e189.

29. Llamosi A, Gonzalez-Vargas AM, Versari C, Cinquemani E, Ferrari-Trecate G, Hersen $\mathrm{P}$, et al. What population reveals about individual cell identity: single-cell parameter estimation of models of gene expression in yeast. PLoS Comput Biol. 2016;12:e1004706.

30. Meir E, von Dassow G, Munro E, Odell GM. Robustness, flexibility, and the role of lateral inhibition in the neurogenic network. Curr Biol. 2002;12:778-86.

31. Leon M, Woods ML, Fedorec AJH, Barnes CP. A computational method for the investigation of multistable systems and its application to genetic switches. BMC Syst Biol. 2016:10:130.

32. Brunel NJ-B. Parameter estimation of ODE's via nonparametric estimators. Electron J Stat. 2008;2:1242-67.

33. Salerno L, Cosentino C, Morrone G, Amato F. Computational modeling of a transcriptional switch underlying B-lymphocyte lineage commitment of hematopoietic multipotent cells. PLoS One. 2015;10:e0132208.

34. van Zelm MC, van der Burg M, de Ridder D, Barendregt BH, de Haas EFE, Reinders MJT, et al. Ig gene rearrangement steps are initiated in early human precursor B cell subsets and correlate with specific transcription factor expression. J Immunol Baltim Md 1950. 2005:175:5912-22.

35. Hucka M, Finney A, Sauro HM, Bolouri H, Doyle JC, Kitano H, et al. The systems biology markup language (SBML): a medium for representation and exchange of biochemical network models. Bioinforma Oxf Engl. 2003;19:524-31.

36. Finney A, Hucka M. Systems biology markup language: level 2 and beyond. Biochem Soc Trans. 2003;31:1472-3.

37. Jolly MK, Jia D, Boareto M, Mani SA, Pienta KJ, Ben-Jacob E, et al. Coupling the modules of EMT and stemness: a tunable "stemness window" model. Oncotarget. 2015;6:25161-74.

38. Huang B, Jolly MK, Lu M, Tsarfaty I, Ben-Jacob E, Onuchic JN. Modeling the transitions between collective and solitary migration phenotypes in Cancer metastasis. Sci Rep. 2015;5 srep17379

39. Bhattacharyya A. On a measure of divergence between two statistical populations defined by their probability distributions. Bull Calcutta Math Soc. 1943;35:99-109.

40. Kullback S, Leibler RA. On information and sufficiency. Ann Math Stat. 1951; 22:79-86.

41. Schaffter T, Marbach D, Floreano D. GeneNetWeaver: in silico benchmark generation and performance profiling of network inference methods. Bioinforma Oxf Engl. 2011;27:2263-70.

42. Margolin AA, Nemenman I, Basso K, Wiggins C, Stolovitzky G, Favera RD, et al. ARACNE: an algorithm for the reconstruction of gene regulatory networks in a mammalian cellular context. BMC Bioinformatics. 2006;7:S7.

43. Klemm K, Bornholdt S. Topology of biological networks and reliability of information processing. Proc Natl Acad Sci U S A. 2005;102:18414-9.

44. Kaluza P, Mikhailov AS. Evolutionary design of functional networks robust against noise. EPL Europhys Lett. 2007;79:48001.

45. Raser JM, O'Shea EK. Noise in gene expression: origins, consequences, and control. Science. 2005;309:2010-3.

46. Munsky B, Neuert G, van Oudenaarden A. Using gene expression noise to understand gene regulation. Science. 2012;336:183-7.

47. Li G, Wang S-W, Rabitz H. Practical approaches to construct RS-HDMR component functions. J Phys Chem A. 2002;106:8721-33.

48. Li G, Hu J, Wang S-W, Georgopoulos PG, Schoendorf J, Rabitz H. Random sampling-high dimensional model representation (RS-HDMR) and orthogonality of its different order component functions. J Phys Chem A. 2006:110:2474-85.

Ready to submit your research? Choose BMC and benefit from

- fast, convenient online submission

- thorough peer review by experienced researchers in your field

- rapid publication on acceptance

- support for research data, including large and complex data types

- gold Open Access which fosters wider collaboration and increased citations

- maximum visibility for your research: over $100 \mathrm{M}$ website views per year

At BMC, research is always in progress.

Learn more biomedcentral.com/submissions 